\title{
Effect of the Electron Beam on the Alloy Properties of Polymethyl Methacrylate and Cycloolefin Copolymer
}

\author{
Maryam Khosravinezhad* and Masumeh Kazemi \\ Department of Polymer Engineering, Faculty of Chemical Engineering, Shiraz branch, Islamic Azad University, \\ Shiraz, Iran \\ * Corresponding author, e-mail: mkhosravi243@yahoo.com
}

Maryam Khosravinezhad: https://orcid.org/0000-0001-6135-3473

Masoumeh Kazemi: https://orcid.org/0000-0001-9756-4096

\begin{abstract}
Polymethyl methacrylate (PMMA) is a transparent thermoplastic with excellent optical properties, transparent surface, low moisture absorption, tensile and electrical resistance. In this study, the alloy prepared through PMMA and cycloolefin copolymer (COC) due to some similar properties. The mechanical test showed that properties such as impact resistance, elongation, tensile, and flexural strength decreased by adding COC up to $20 \%$ due to less incompatibility and miscibility, but mentioned properties improved by adding COC $40 \%$ due to sub-phase generation. The DSC and DMTA tests showed improvement in the thermal properties of alloy by adding 40\% COC. SEM micrographs exhibited a softer surface and more phase elongation of the alloy. Finally, the sample selected as the optimal sample in terms of mechanical properties irradiated by electron beam, and amplification results showed that a dose of $50 \mathrm{KGY}$ increased the mechanical and thermal properties relatively.
\end{abstract}

Keywords: Polymethyl methacrylate, Cycloolefin copolymer, Mechanical test, Electron Beam

\section{Introduction}

Polymethyl methacrylate is one of the most well-known polymers that are widely used in the glass industry [1]. This material is one of the hardest and most important polymers with glass transparency, glossy, polished, and weather-resistant surface. PMMA sheets have significant resistance to atmospheric agents and sunlight [2-4]. The high price of PMMA has led to the use of cheap polymers or various copolymers to make alloys. Copolymers are created by the polymerization of two or more suitable monomers, which lead to distinct structures. In this regard, it can refer to COC which has also glass-like transparency [5], low density [6], temperature deviation [7], electrical properties [8], high tensile modulus [9], and suitable hardness [10]. This copolymer showed a low moisture absorption capacity, resistant to acids, and acts as an organic pole of chemicals. In polyolefin mixtures, COC results in a higher modulus and heat resistance and is often used as a component to enhance the modulus and reduce the film thickness.

Mixing of some polymers leads to a miscible alloy. The mechanical properties of miscible alloys follow the reaction of the blends or show a positive deviation $[11,12]$. Due to the small entropy and 
positive enthalpy of blending, most alloys are immiscible and therefore show a multiphase morphology. The mechanical properties are strongly influenced by the morphology of the phases and the properties of the interface [13]. Also; morphology is influenced by thermodynamic parameters, rheology, and process conditions. Rheological parameters such as component viscosity have a significant effect on the phase structure of the blend. On the other hand, the rheological behavior of the alloy depends on the type of morphology and the interface between the phases. Information which obtained from morphological studies can be useful in predicting and mechanical properties of alloys. Over the past few decades, research has focused on modifying existing polymers to create new materials with desirable properties [14-16].

High-energy radiation can be applied to network polymers $[17,18]$ and the cobalt radiation is appropriate from electronic generators. The radiation from these systems consists of alpha, beta, and gamma rays with different frequencies. Irradiation of ionizing rays on polymeric materials leads to the reactions, ion formation, free radicals, and consequently, changes in the chemical structure of polymers. Even a small chemical change in the molecular chain leads to an impact on the polymer behavior. This effect can be observed in the final physical-mechanical and even biological properties. Polymer alloy irradiation is the new field of polymer irradiation, which is used to stabilize the morphology of alloy or compatibility between the two components and has a high potential to play a commercial role. Lee et al. investigated the failure and crosslinking mechanism of PMMA, which exposed to different radiation sources. The results show that both failures and crosslinking occur during irradiation. LET (linear energy transfer) electronic and nuclear energy cause networking and chain failure, respectively [19]. According to Ravindrachary et al.'s report, the microstructural study on electron irradiated PMMA, it was concluded that the presence of carbon clusters as a result of chain failure in PMMA was due to radiation [20]. Few studies have been performed on the PMMA and COC alloys, under irradiation conditions.

Since PMMA and COC are polar and non-polar copolymers, respectively, it is expected that the two materials will not be compatible with each other, but crosslinking may occur by irradiation, and some properties of the alloy maybe improved. Many aspects make cross-linked PMMA superior such as the exceptional mechanical properties, great chemical and abrasion resistance [21, 22]. Therefore, due to the non-polarity of $\mathrm{COC}$, this study aimed to determine the compatibility, relationship between different percentages of copolymer and the mechanical properties, and to optimize the price of the alloy.

\section{Materials and Methods}

In this research, PMMA (grade CM-205) with melt flow index of min $1.8 \mathrm{~g} / 10 \mathrm{~min}$ from CHI MEI Taiwan, and COC were purchased from TOPAS Germany (grade 6015).

\subsection{Samples Preparation}


In the first step, PMMA and COC were mixed manually and alloy samples were prepared by combining the percentages presented in Table (1). Thus, PMMA and COC were dried in a thermal oven for 6 hours at $80^{\circ} \mathrm{C}$ and alloying was performed using an extruder with two identical round screws. The process temperature and speed was $230-260^{\circ} \mathrm{C}$ and $200 \mathrm{rpm}$. A vacuum pump was also placed in the extruder to remove residual air during alloying. The extruded specimens were granulated and the dumbbell specimens prepared for tensile, bending, and impact tests according to ASTM standard with KRAUSSMAFFEI KM injection molding machine. For all samples, mold temperature and injection pressure were $70^{\circ} \mathrm{C}$, and $30 \mathrm{MPa}$. Finally, the samples were dried in a vacuum oven for 2 hours, and stored in a dry place at an ambient temperature of about $27 \pm 2{ }^{\circ} \mathrm{C}$.

\subsection{Tensile Test}

The dimensions of the sample were first accurately measured with a caliper. The stress by dividing the force to the surface, and the strain by dividing the displacement to the initial length was obtained and the stress-strain curve was drawn. The material behavior was determined through the stress-strain curve.

\subsection{Three-Point Bending Test}

One of the most common bending module tests is the three-point bending test. The samples were placed on two supports, and force was applied from the above and middle of the two supports. In this case, the lower surface of the sample was stretched, and the upper surface was pressed so that if the compressive and tensile modulus of the specimens were equal, the stress in the middle axis of the specimen would be zero.

\subsection{Impact Test}

Impact test was performed by GOTECH impact machine model TCS2000 (Taiwan) according to standard (ASTM D256). The amount of energy consumed by breaking a standard-sized specimen was obtained by hitting a standard hammer. The impact resistance was calculated by dividing the energy to the cross-sectional area or the sample thickness.

\subsection{Differential Scanning Calorimetry (DSC)}

In this study, the Shimadzu 50 -DSC device was used for DSC determination. According to the International Confederation of Scanning Thermal Analysis, two samples that were subjected to the same heating or cooling rate were used to show the temperature difference between the reference sample and the sample, which is a function of temperature or time. The calibration was performed based on the heat dissipation reaction of a suitable standard material.

\subsection{Gelling Test}


Toluene solvent was used to measure the gel content of the samples by extraction method. The samples were placed in the desired solvent for 24 hours and then dried in an oven at a temperature of $50{ }^{\circ} \mathrm{C}$ to reach a constant weight.

\subsection{Investigation of Dynamic Mechanical-Thermal Behavior}

The PL-DMTA MKII DMTA device was used to determine the transfer temperatures, especially the glass transfer temperature. Dynamic mechanical thermal analysis (DMTA) was applied to study the $\mathrm{Tg}$ transfer temperatures as well as the storage modulus, the loss modulus in polymers, and to study dynamic mechanical properties over a wide temperature range.

\subsection{Scanning Electron Microscope (SEM)}

SEM was relatively faster and had a higher resolution than other methods. In SEM, an image was formed on a television screen by traversing an electron beam focused on the surface of a sample under a vacuum.

\subsection{Electron Beam Test}

In this project, the samples were irradiated using a TT200 Rhodotron electron accelerator. It was a Rhodotron accelerator with four vertical and horizontal outputs and energies of 5 and 10 million electron volts, and the final power of this device was $100 \mathrm{~kW}$, which could be increased by double.

\section{Results and Discussion}

\subsection{Tensile Test}

Tensile diagrams of alloys (Figure 1) showed that with increasing COC from 5 to 20\%, tensile strength and elongation decreased, but these two factors increased using $40 \%$. The reduction of tensile strength and elongation parameters can be found in the incompatibility of the two polymers. The tensile strength and elongation of PMMA were higher than COC. In the alloy containing 5\% COC, PMMA as the dominant phase determined the properties of the alloy. The increase in COC sub-phase, the properties of alloys decreased due to structural similarity of polymers. It was observed that in the alloy containing $40 \% \mathrm{COC}$, the tested properties in turn were increased again. Presumably, the presence of ethylene group in the COC structure caused increasing in PMMA flexibility. The subphase, which made up $40 \%$ of the alloy, was able to exhibit its properties in the second phase.

Figure 2 revealed that both failure and crosslinking occurred during electron beam, but the crosslinking and failure ratio depended on the polymer structure. The composition and structure of PMMA changed significantly in volatile moieties, such as hydrogen, after exposure to high doses. But the COC became somewhat networked with increasing radiation dose. At the KGY 25 dose, the tensile strength decreased, and the crosslinking did not change, but the tensile strength increased at the KGY 50 dose, relatively. 


\subsection{Bending Test}

Figure 3 showed the bending test of PMMA/COC alloy with weight percentages of 5, 10, 20, and 40 . The bending test confirmed the results of the tensile test. Flexural strength decreased with increasing COC to $20 \%$. But the flexural strength increased at the $40 \%$ level. The rise of phase COC and the increase of the knot caused the flexural strength. Figure 4 showed the results of PMMA/COC40 alloy after irradiation at different doses. The results showed that irradiation at a dose of 50 improved the flexural strength, and other doses used did not have a significant effect. The use of radiation (dose of 50) created enough crosslinks. Other doses increased brittle behavior.

\subsection{Impact Test}

In this study, the impact test of non-notch specimens was measured in Izod impact strength test. For each alloy, three replications were considered and the average of each alloy was used to plot the graph (Figure 5). The impact strength of the alloy decreased with rising COC up to 20\%. This decrease was due to the incompatibility of two polymers and the lack of proper mixing. Also, the PMMA phase, which had a lower impact on strength, determined the alloy properties. But the impact resistance increased with the addition in COC level to $40 \%$. This compatibility appeared to be due to the proper networking of the COC alongside the PMMA (matrix). Figure 6 showed the impact test results of PMMA / COC40 irradiated alloy. The strength of the alloy was improved using a 50-radiation dose. Impact strength showed a significant decrease with increasing radiation dose due to occupy of radical hydrogens in higher irradiation doses.

\subsection{Differential Scanning Calorimetry Test (DSC)}

The DSC results of the best (PMMA/COC40) and worst (PMMA/COC20) alloy are showed in Figure 7. The Tg ratio of the PMMA/COC20 and PMMA/COC40 (Figure 7) showed that COC and PMMA in the PMMA/COC40 alloy were the same and occurred approximately in the temperature range 115 and $135^{\circ} \mathrm{C}$. But in PMMA/COC20 alloy, the phases were slightly separated from each other (PMMA at 123 and $\mathrm{COC}$ at $143^{\circ} \mathrm{C}$ ). The destruction of the three-dimensional COC networks caused closer Tg peaks at 50 than 100 doses. As the COCs crosslinked, slight coherence caused the $\mathrm{Tg}$ of the material to converge. Also, the heating rate of KGY100 irradiated alloy was lower than other alloys.

\subsection{Dynamic Mechanical Thermal Analysis (DMTA)}

The viscoelastic properties in Figure 9 showed that $\mathrm{Tg}$ of COC and PMMA overlapped in the PMMA/COC40 alloy. However, there was relatively little homogeneity between PMMA and COC in the PMMA/COC20. Therefore, two Tg peaks were observed in the viscoelastic curve. PMMA/COC40, which confirmed the modulus of dissipation and stress dissipation, was more than $20 \%$, and an alloy containing 40\% COC can eliminate the stress (Figure 9). The Tg in Figures 9 and 10 exhibited, PMMA and COC increased at a higher rate of 50-dose irradiation due to a threedimensional network. However, with increasing the dose from 50 to $100 \mathrm{KGY}$, due to the destruction 
of the chains and the reduction of the three-dimensional COC network, the Tgs of both phases decreased again to lower values In both 50 and 100 doses, two peaks due to low Crosslink and threedimensional lattice were showed to separate the phases.

\subsection{Scanning Electron Microscope (SEM)}

The SEM (Figures 11) of PMMA/COC40 was used to study the morphology of the sample. Due to the incompatibility of COC in PMMA, COC particles were dispersed as a separate sub-phase within the matrix, while the reduction of the surface energy of COC caused a spherical state. The PMMA/COC40 had a softer structure than the PMMA/COC20 and clearly showed phase elongation and failure tolerance during impact and stress. Increasing the COC sub-phase resulted in more solubility of COC in the PMMA matrix, greater homogeneity, and a softer surface and allowed the particles to form a continuous sub-lattice within the matrix. PMMA/COC40 in the irradiated and nonirradiated state concluded that (irradiation and electron beam at $50 \mathrm{KGy}$ ), the COC particles were somewhat out of spherical shape, which indicated the low crosslink that caused increased strength and eventually "energy" failed. The high energy input, the breakage of the polymer chains, and the rebreaking of the three-dimensional network identified by the white powder particles by increasing the radiation dose from 50 to $100 \mathrm{KGy}$. The results of this study are consistent with Ogilvie et al. [23].

\subsection{Crosslink Test of PMMA/COC4O}

Table 2 and Figure 12 showed that the rising radiation dose increased the cross-links due to the crosslinking of COC and PMMA. Increasing the radiation dose up to $50 \mathrm{KGy}$ improved the crosslinking properties, but the gelling properties decreased significantly, and the PMMA structure became prone to degradation.

\section{Conclusion}

This study aimed to prepare a two-component alloy of PMMA and COC. Cyclic olefin copolymer was used to improve the properties of PMMA. The results of tensile test showed that at relatively low percentages of $\mathrm{COC}$, tensile strength decreased due to incompatibility, but with increasing percentage of COC, tensile strength increased due to dominance of this phase. Three-point bending test showed that in the sample containing $40 \%$ COC, the mechanical properties improved. The dose of KGY 25 irradiation had little effect on the sample, but with increasing the dose to KGY50 improved the mechanical properties. Through DMTA and DSC tests, it was observed that the alloy containing 60\% PMMA and $40 \%$ COC had the best properties.

\section{Conflicting Interests}

The author(s) declared no potential conflicts of interest with respect to the research, authorship, and/or publication of this article.

\section{Authors' Contributions}


M. Kazemi has the same contribution as the first author.

\section{Funding}

The author(s) received no financial support for the research, authorship, and/or publication of this article.

\section{References}

1. Zhang, Y., Liu, W., Huang, W., Ding, Y., Song, L., Zheng, S. and Wang, Z., "The toughening of polymeric glasses using cellulose without sacrificing transparency", Industrial Crops and Products, 142, p.111842, 2019.

2. Gallo, Leonardo Sant'Ana, Mariana OC Villas Boas, Ana CM Rodrigues, Francisco CL Melo, and Edgar D. Zanotto. "Transparent glass-ceramics for ballistic protection: materials and challenges." Journal of Materials Research and Technology 8, no. 3, 3357-3372, 2019.

3. Zidan, H. M., and M. Abu-Elnader. "Structural and optical properties of pure PMMA and metal chloride-doped PMMA films." Physica B: Condensed Matter 355, no. 1-4, 308-317, 2005.

4. Bafna, Minal, Ankit K. Gupta, Abhinandan Agarwal, Neha Sain, and Vipin Jain. "Insight into the optical properties of poly-methyl-methacrylate (PMMA) loaded with ferric chloride $(\mathrm{FeCl} 3)$ in varying concentration." Materials Today: Proceedings (2020).

5. Lu, Jin, Yuanbao Qiang, Wangqing Wu, and Bingyan Jiang. "Experimental study on viscosity properties of cyclic olefin copolymer (COC) flowing through micro capillary dies." Polymer Testing 89, 106635, 2020.

6. Vacková, Tat’ana, Miroslav Slouf, Martina Nevoralová, and Ludmila Kaprálková. "HDPE/COC blends with fibrous morphology and their properties." European polymer journal 48, no. 12, 2031-2039, 2012.

7. Zhang, Ruiyan, Eric S. Kim, Sandra Romero-Diez, Yaxian Wang, Gang Huang, Andy Li, Yong Yang, and Patrick C. Lee. "Cyclic olefin copolymer foam: A promising thermal insulation material." Chemical Engineering Journal 409, 128251, 2021.

8. Kasgoz, Alper, Dincer Akın, and Ali Durmus. "Rheological and electrical properties of carbon black and carbon fiber filled cyclic olefin copolymer composites." Composites Part B: Engineering 62, 113-120, 2014.

9. Pegoretti, Alessandro, Jan Kolarík, Luca Fambri, and Amabile Penati. "Polypropylene/cycloolefin copolymer blends: effects of fibrous phase structure on tensile mechanical properties." Polymer 44, no. 11, 3381-3387, 2003.

10. Kasgoz, Alper, Dincer Akın, Ali Imran Ayten, and Ali Durmus. "Effect of different types of carbon fillers on mechanical and rheological properties of cyclic olefin copolymer (COC) composites." Composites Part B: Engineering 66, 126-135, 2014. 
11. Mohanty, Subhra, Golok B. Nando, K. Vijayan, and N. R. Neelakanthan. "Mechanical and dynamic mechanical properties of miscible blends of epoxidized natural rubber and poly (ethylene-co-acrylic acid)." Polymer 37, no. 24, 5387-5394, 1996.

12. Prinos, J., Ch Tselios, D. Bikiaris, and C. Panayiotou. "Properties of miscible blends of polyglutarimide with poly (styrene-co-maleic anhydride)." Polymer 38, no. 24, 5921-5930, 1997.

13. Hou, Harvey JM, and David Mauzerall. "Listening to PS II: Enthalpy, entropy, and volume changes." Journal of Photochemistry and Photobiology B: Biology 104, no. 1-2, 357-365, 2011.

14. Wu, Guosong, Jamesh Mohammed Ibrahim, and Paul K. Chu. "Surface design of biodegradable magnesium alloys_-a review." Surface and Coatings Technology 233, 2-12, 2013.

15. Takeichi, Tsutomu, Shoya Uchida, Yuichi Inoue, Takehiro Kawauchi, and Nobuyuki Furukawa. "Preparation and properties of polymer alloys consisting of high-molecular-weight benzoxazine and bismaleimide." High Performance Polymers 26, no. 3, 265-273, 2014.

16. Wang, Rui, Jin Yu, Juntao Tang, Ruijin Meng, Linda F. Nazar, Lezhi Huang, and Xiao Liang. "Insights into dendrite suppression by alloys and the fabrication of a flexible alloy-polymer protected lithium metal anode." Energy Storage Materials 32, 178-184, 2020.

17. Rouif, Sophie. "Radiation cross-linked polymers: Recent developments and new applications." Nuclear Instruments and Methods in Physics Research Section B: Beam Interactions with Materials and Atoms 236, no. 1-4, 68-72, 2005.

18. Šarac, T., N. Quiévy, A. Gusarov, and M. J. Konstantinović. "The study of temperature and radiation induced degradation of cable polymers: A comparison between the mechanical properties of industrial and neat EPDM." Procedia Structural Integrity 2, 2405-2414, 2016.

19. Lee, E. H., G. R. Rao, and L. K. Mansur. "LET effect on cross-linking and scission mechanisms of PMMA during irradiation." Radiation physics and chemistry 55, no. 3, 293-305, 1999.

20. Ravindrachary, V., Rajashekhar F. Bhajantri, S. D. Praveena, Boja Poojary, Dhanadeep Dutta, and P. K. Pujari. "Optical and microstructural studies on electron irradiated PMMA: A positron annihilation study." Polymer Degradation and Stability 95, no. 6, 1083-1091, 2010.

21. Min K, Silberstein M, Aluru NR. Crosslinking PMMA: Molecular dynamics investigation of the shear response. Journal of Polymer Science Part B: Polymer Physics. 2014; 52(6):444-9. https://doi.org/10.1002/polb.23437

22. Albeladi, H. K., A. N. Al-Romaizan, and M. A. Hussein. "Role of cross-linking process on the performance of PMMA." Int. J. Biosens. Bioelectron 3, 279-284, 2017.

23. Ogilvie, I. R. G., V. Sieben, C. F. A. Floquet, R. Zmijan, M. Mowlem, and H. Morgan. "Solvent processing of PMMA and COC chips for bonding devices with optical quality surfaces." In 14th international conference on miniaturized systems for chemistry and life sciences, pp. 1244-1246. 2010. 


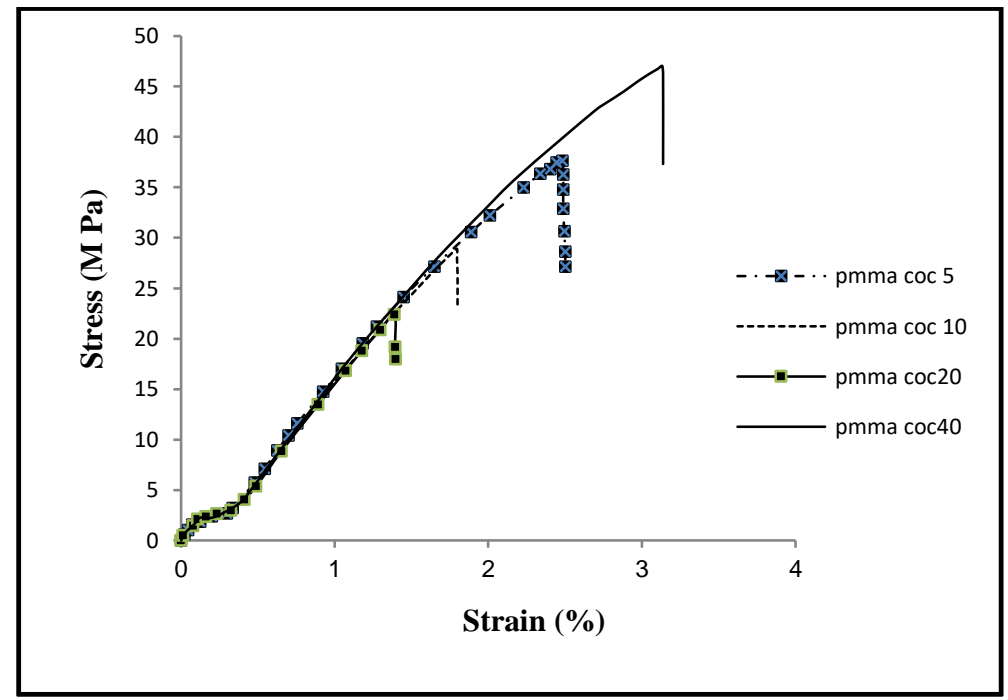

Figure 1 Results tensile test of PMMA/COC alloy with weight percentages of 0, 10, 20 and 40 of COC

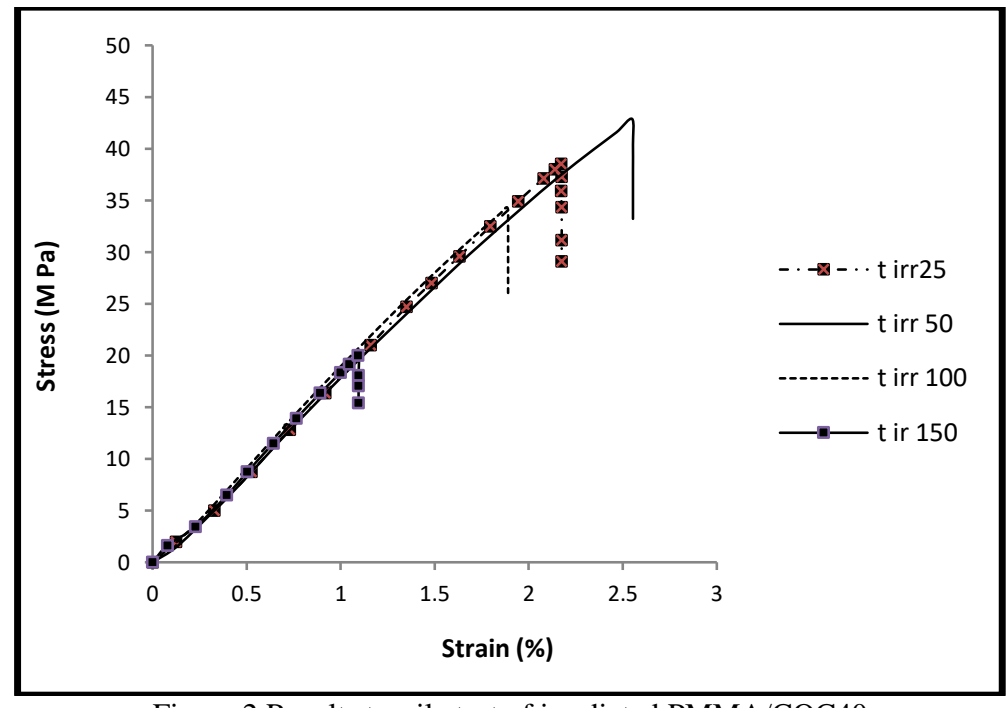

Figure 2 Results tensile test of irradiated PMMA/COC40 


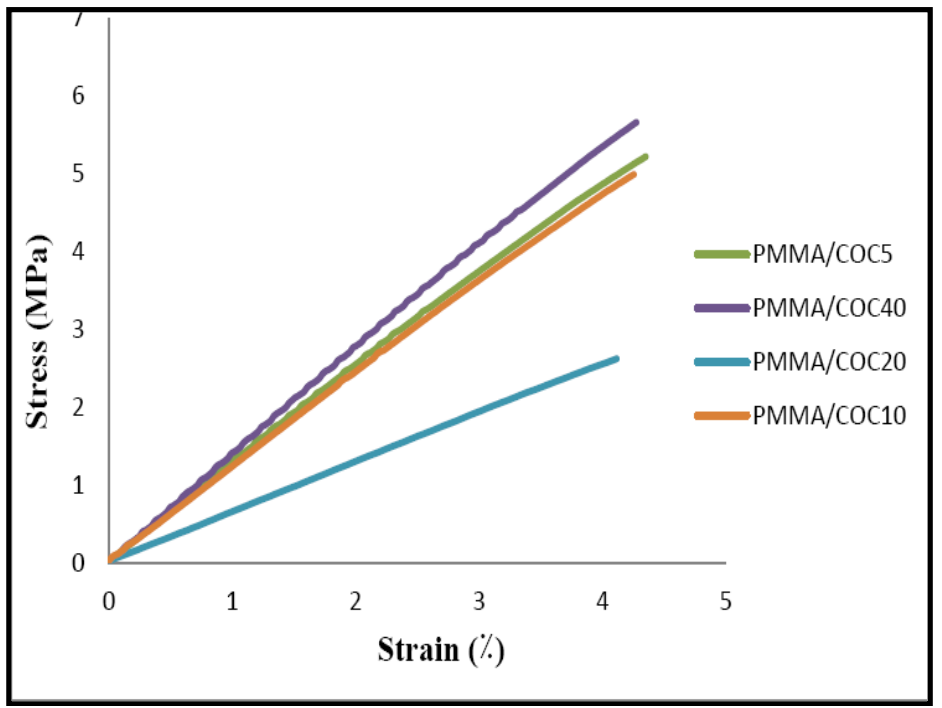

Figure 3 Results bending test of PMMA/COC alloy with weight percentages of 0, 10, 20 and 40 of COC

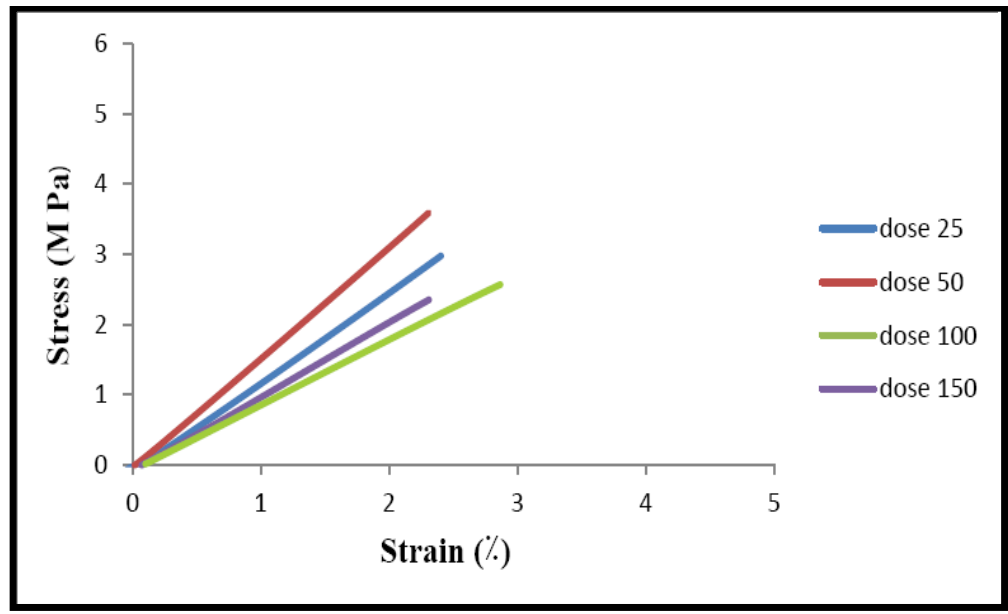

Figure 4 Results bending test of irradiated PMMA/COC40 


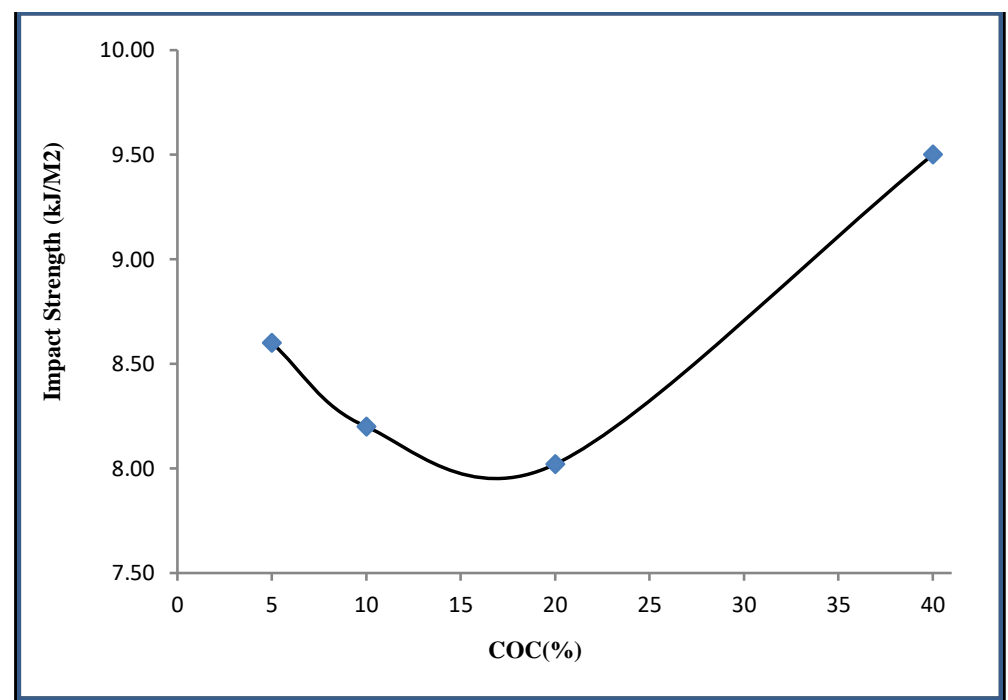

Figure 5 Results impact test of PMMA/COC alloy with weight percentages of 0, 10, 20 and 40 of COC

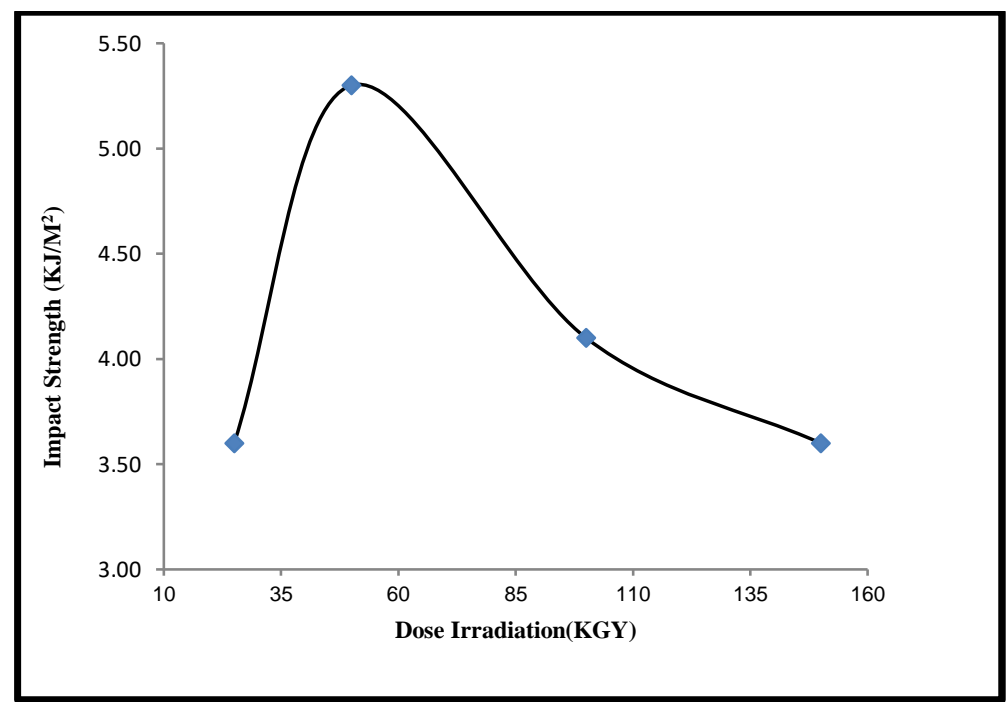

Figure 6 Results impact test of irradiated PMMA/COC40 


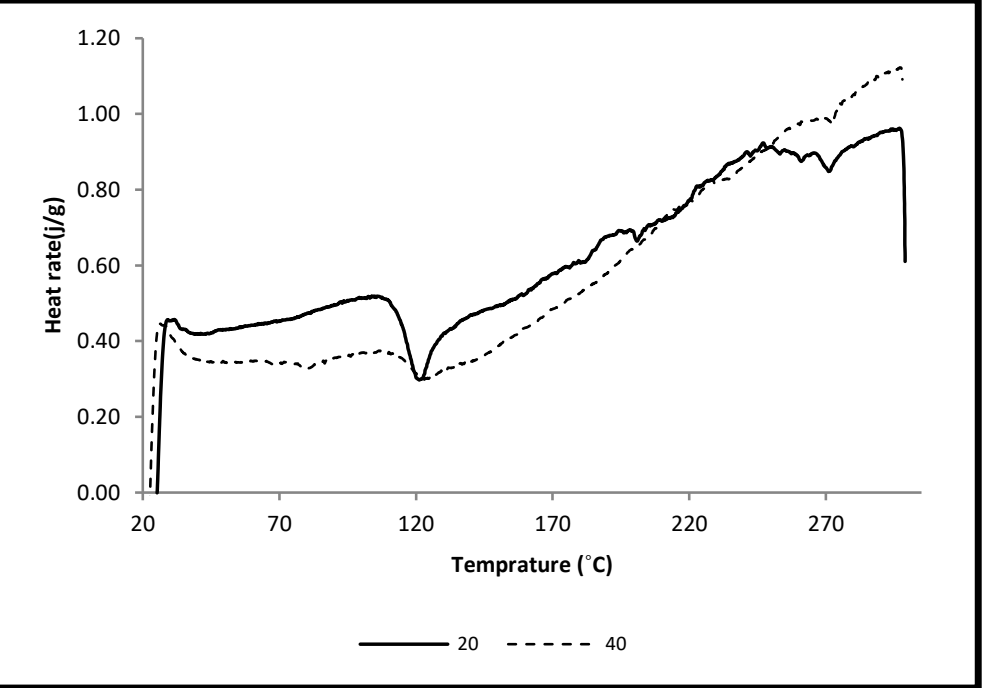

Figure 7 DSC test of PMM /COC20 and PMMA/COC40 samples before irradiation

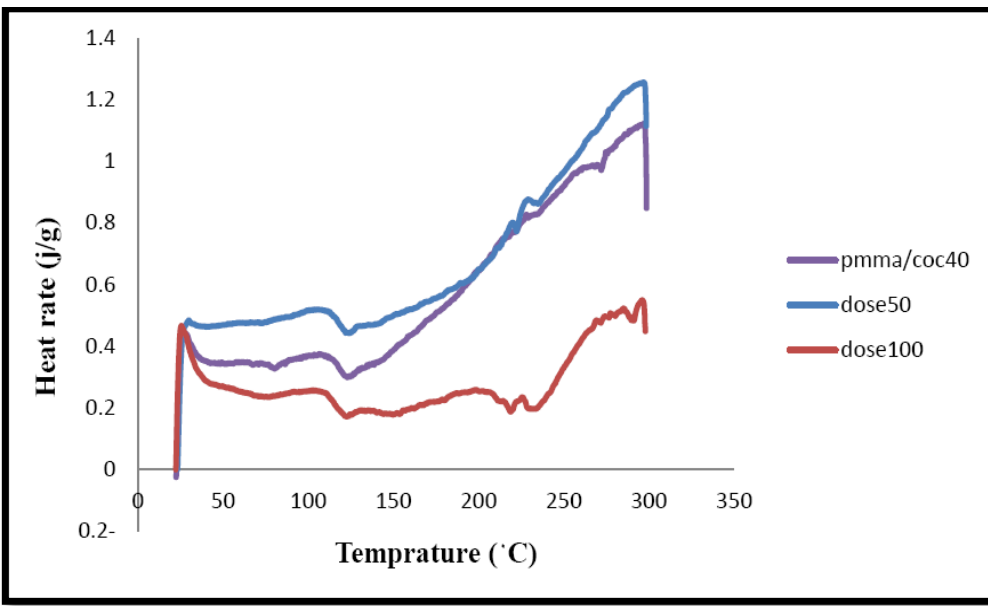

Figure 8 DSC test of PMMA/COC40 sample after irradiation at 50 and 100 doses (KGY)

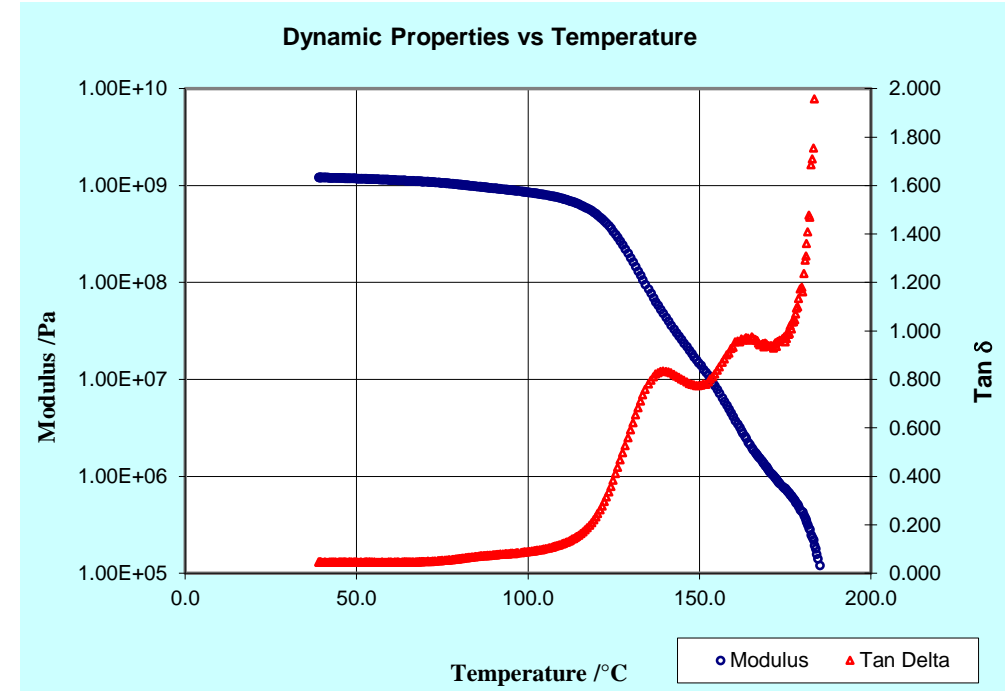

Figure 9 DMTA test of PMMA/COC40 sample after irradiation at 50 doses (KGY) 


\section{- Modulus $\triangle$ Tan Delta Dynamic Properties vs Temperature}

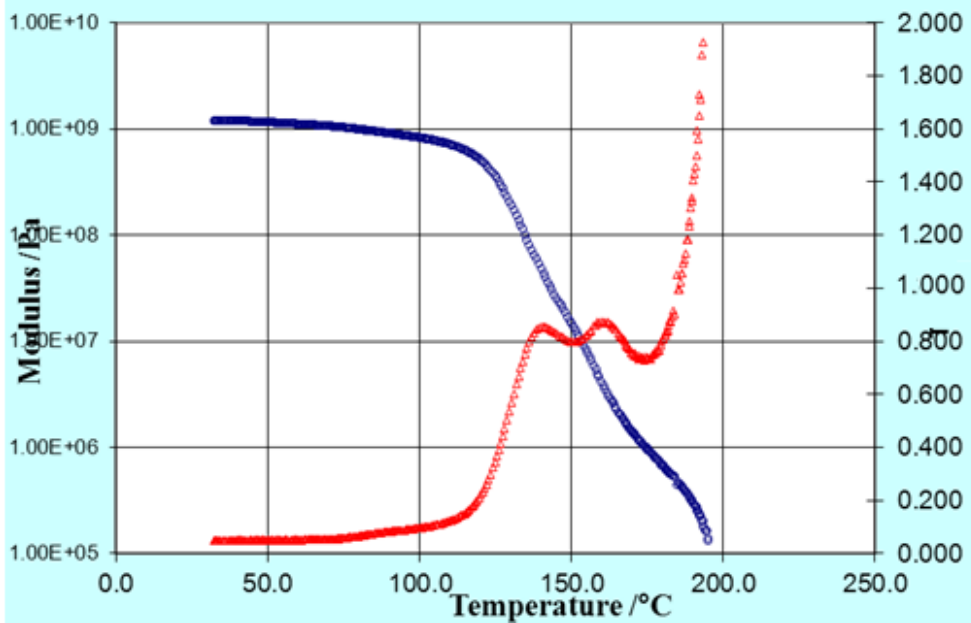

Figure 10 DMTA test of PMMA/COC40 sample after irradiation at 100 doses (KGY)

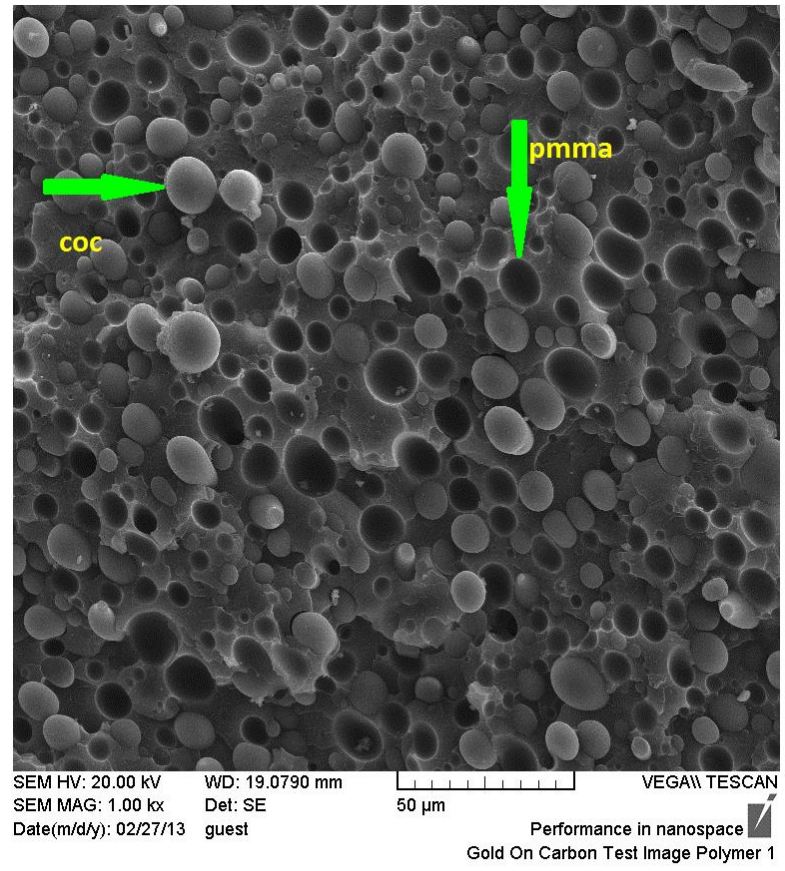

PMMA/COC20

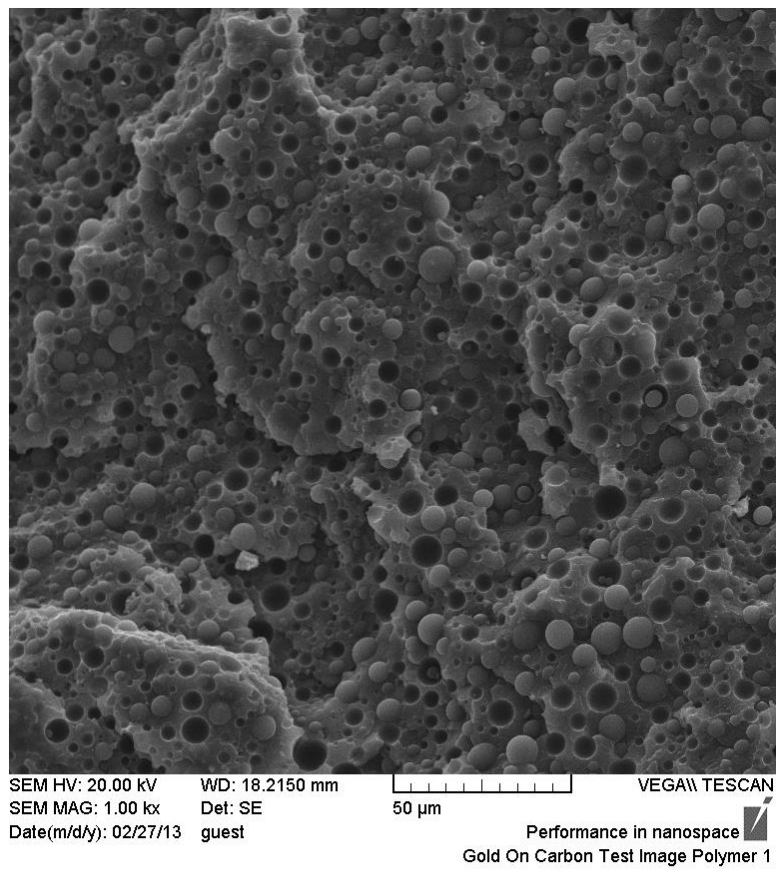

PMMA/COC40

Figure 11 SEM images of PMMA/COC20 and PMMA/COC40 with 1000X magnification (The white spherical particles and the matrix were COC and PMMA, respectively) 


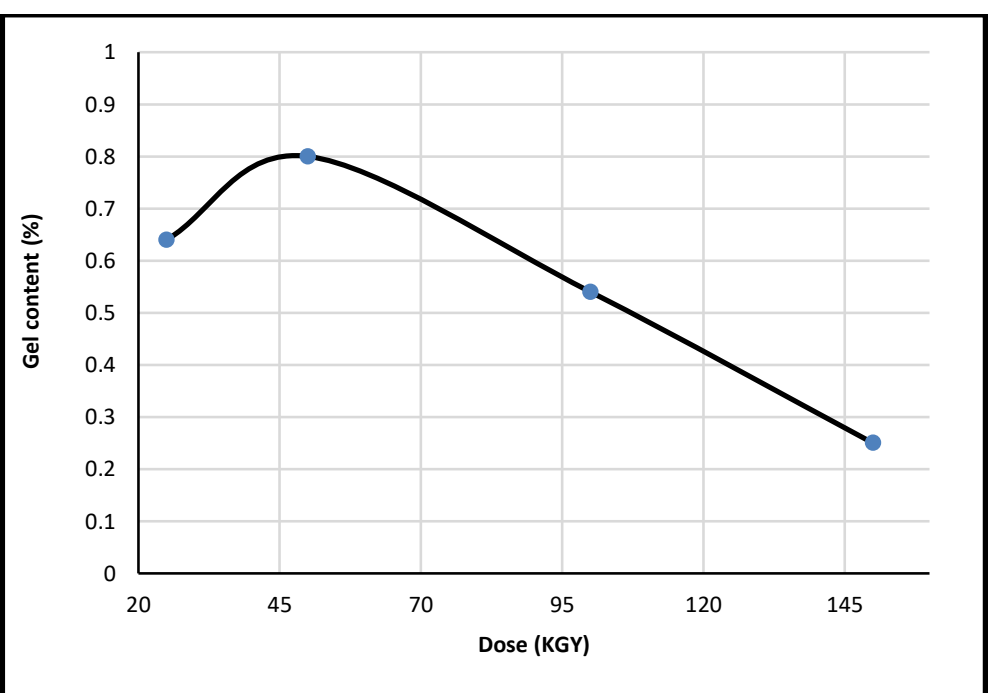

Figure 12 Gel content PMMA/COC40

Table 1 Percentage of compounds in alloy samples

\begin{tabular}{ccc}
\hline Sample & PMMA & COC \\
\hline PMMA/COC $_{5}$ & 95 & 5 \\
PMMA/COC $_{10}$ & 90 & 10 \\
PMMA/COC $_{20}$ & 80 & 20 \\
PMMA/COC $_{40}$ & 60 & 40
\end{tabular}

Table 2 Gel percentage test results of COC, PMMA and PMMA/COC40

\begin{tabular}{cccc}
\hline Electron radiation dose & COC & PMMA & PMMA/COC40 \\
\hline 25irr & 0.78 & 0.50 & 0.64 \\
50irr & 1.00 & 0.60 & 0.80 \\
100irr & 0.70 & 0.30 & 0.54 \\
150irr & 0.30 & 0.20 & 0.25
\end{tabular}

